\title{
HERA has Been Closed, LHC is Being Opened: Near Past and Near Future of Particle Physics
}

\author{
Carlos A. García Canal \\ Laboratorio de Física Teórica, Departamento de Física \\ Universidad Nacional de La Plata, C.C.67, La Plata (1900), Argentina
}

(Received on 15 June, 2008)

\begin{abstract}
A general account of the experimental results of the recently closed DESY-HERA accelerator and a presentation of the physics expected to be revealed by the LHC-CERN accelerator, beginning to deliver data in less than one year, is provided. An analysis of the impact of this information on the theoretical developments is included.
\end{abstract}

Keywords: Standard Model; Supersymmetry; Lattice QCD

We start by reproducing two recent press releases directly connected with the title of our lecture and that motivated it:

On June 30, 2007, data taking at the electron-proton storage ring HERA at DESY will come to an end: For 15 years the particle physics experiments at HERA have provided a unique and detailed picture of the proton and the interacting forces said Prof. Rolf-Dieter Heuer, Research Director at DESY.

Geneva, 22 June 2007: Speaking at the 142nd session of the CERN Council today, the organization's director general Robert Aymar announced that the Large Hadron Collider (LHC) will start up in may 2008, taking the first steps towards studying physics at a new high-energy frontier.

Today it is certainly accepted that Fundamental Symmetry is a synonym of Particle Physics. This assertion has already its origin in the Relativity of Einstein. In fact, Wigner put it clearly in his phrase: "Einstein's work marks the inversion of a tendency, formerly the invariance principles were obtained from the laws of movement. Now it is natural for us to obtain the laws of Nature and prove their validity by means of the invariance laws.

The present engine of development in Physics is Gauge Symmetry, that provides a unified treatment of the fundamental forces. The Standard Model of strong, weak and electromagnetic interactions, that we call (for obvious reasons) 3 ! is based on the symmetry $S U(3) \otimes S U(2) \otimes U(1)$. In 't Hooft words it is the "Temporary solution to nearly a century of questions". 3 ! is one of the greatest success of physics. It comprises practically all that is known of the subatomic world in a concise set of principles and equations. Moreover, it exceeded experimental tests in energy ranges very distant from the ones included in its original design.

The present knowledge of Quantum Field Theory based on gauge symmetry includes the important running behavior of coupling constants. Namely, the value of interaction couplings depends on the momentum scale that one is exploring. This is clearly seen in Fig. 1 for the case of Quantum Chromodynamics (QCD $\equiv$ the 3 in 3 !).

There is a general consensus on the fact that 3 ! is not complete. It has aesthetical deficiencies and there are phenomena that are not included in it. Let us just mention some open questions: which is the cause of the symmetry breaking?; how do gauge interactions unify?; and Gravity?; what about dark matter and dark energy?; is there something else?; etc.

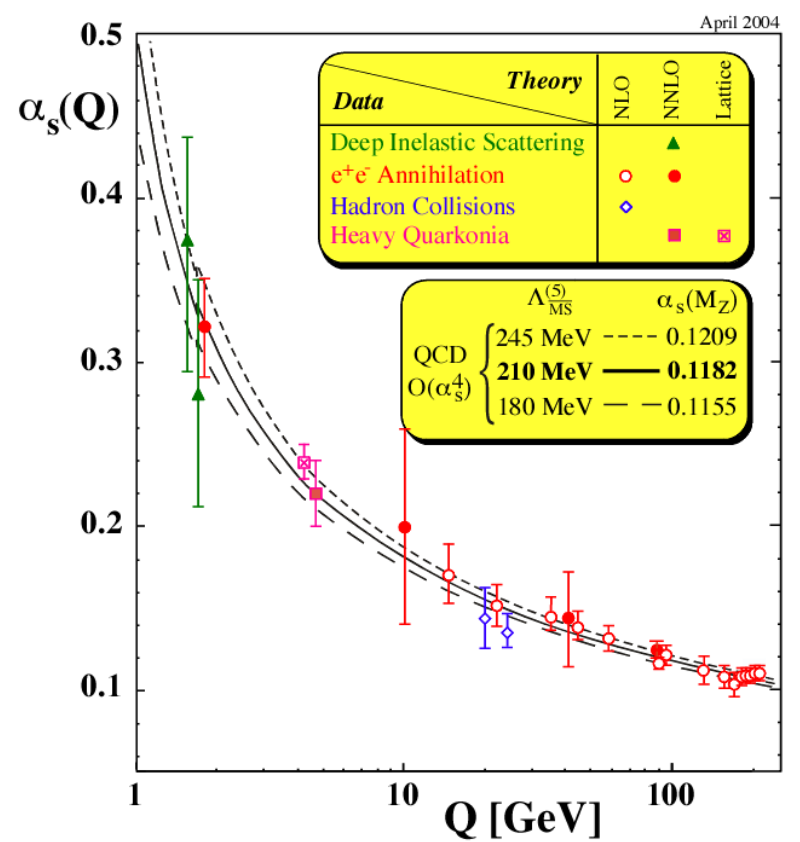

FIG. 1: QCD running coupling constant

In order to answer these and other important questions, high energy collider accelerators have been and are the principal tools. In these machines, beams of $e^{-}, e^{+}, p, \bar{p}$ are accelerated in opposite directions and collide frontally. The next point is to decide in favor of electron or (and) protons and we are going to briefly discuss on it.

Let us start by considering electron colliders. The paradigm of a recent collider of this type was LEP (Large Electron Positron) at CERN. In this case, being the electron elementary, the initial state is well defined. On the contrary, the cross section $\sigma\left(e^{-} e^{+}\right)$is small (electromagnetic interaction) and being the electron mass very small, the corresponding synchroton radiation is very important. For this reason, LEP needed a length of around $27 \mathrm{Km}$ in order to accelerate electrons above $100 \mathrm{GeV}$. LEP was really the Standard Model machine because it was tuned around the $Z^{0}$ mass. In fact, around 20 millons of $Z^{0}$ were produced and analyzed. This very large statistics allowed a very precise determination of the $Z^{0}$ parameters (mass, width, coupling to fermions, asymmetries, etc.)

One of the very nice results obtained at LEP showing that 


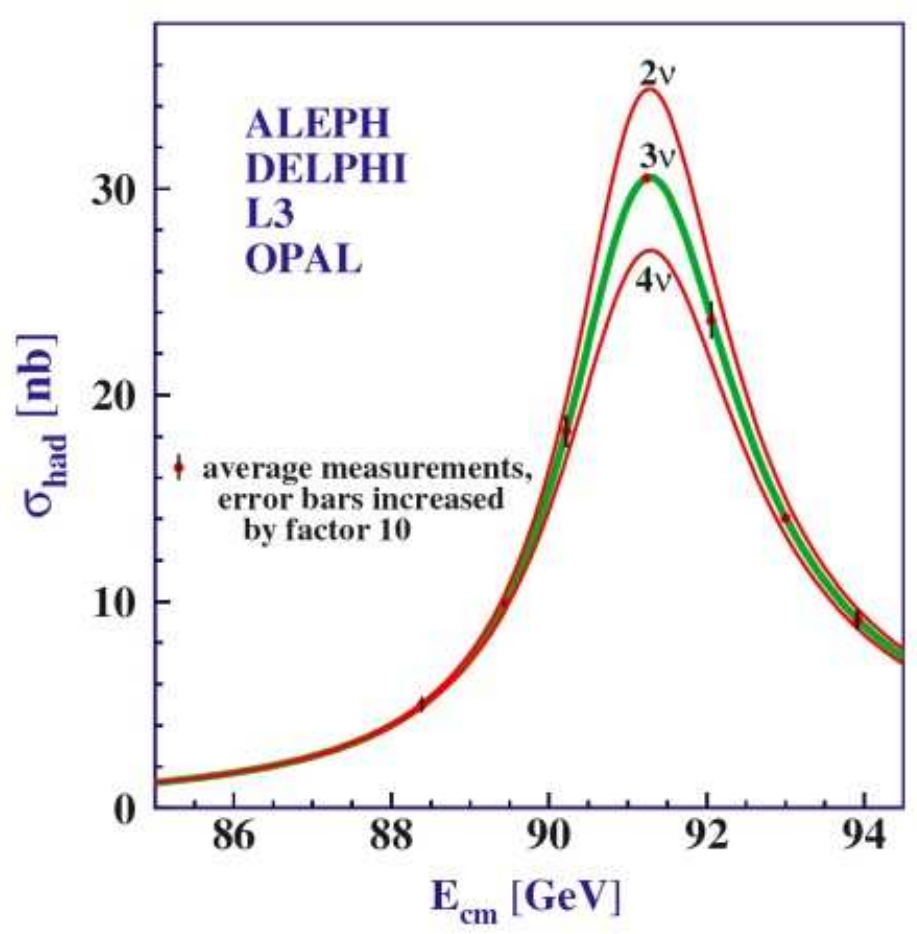

FIG. 2: Number of families

the number of families (number neutrino flavors with mass smaller than half the $Z^{0}$ mass) is presented in Fig.2

Other quite impressive result is related to the experimental evidence of the coupling among gauge bosons, as shown in Fig. 3 where experimental data on $W^{+} W^{-}$production is presented together with the prediction including neutrino, photon and $Z^{0}$ intermediate states.

Let us go now to analyze the proton (-antiproton) collider. We refer to the TEVATRON at FERMILAB. In this case the synchroton radiation is not important. For this reason here the length of the accelerator is only $6 \mathrm{Km}$ to attain proton energies of $1 \mathrm{TeV}$. Moreover, the cross section, due to strong interactions, is large and consequently one obtains many events. The problem is that protons are composite particles and the collision is in fact among proton components that share the proton momentum.

TEVATRON allowed also a detailed study of the 3!, in particular the physics of the $B-$ mesons, the analysis of QCD (the 3 of 3 !) and the electroweak processes, the search for the Higgs boson, etc. Among the specific result we should mention the increasing in precision in the determination of the $W$ mass, the study of QCD with photons and jets, the CabbiboKobayashi-Maskawa mixing matrix and the most resonant achievements related to the top quark discovery and the detection of $C P$ violation in the $B-$ meson sector.

This brief racconto of the near past puts in evidence that electron colliders and proton colliders are complementary. The electron (positron) version provides clean experiments and consequently precision, while the proton version provides larger energies for discoveries and consequently potentiality.

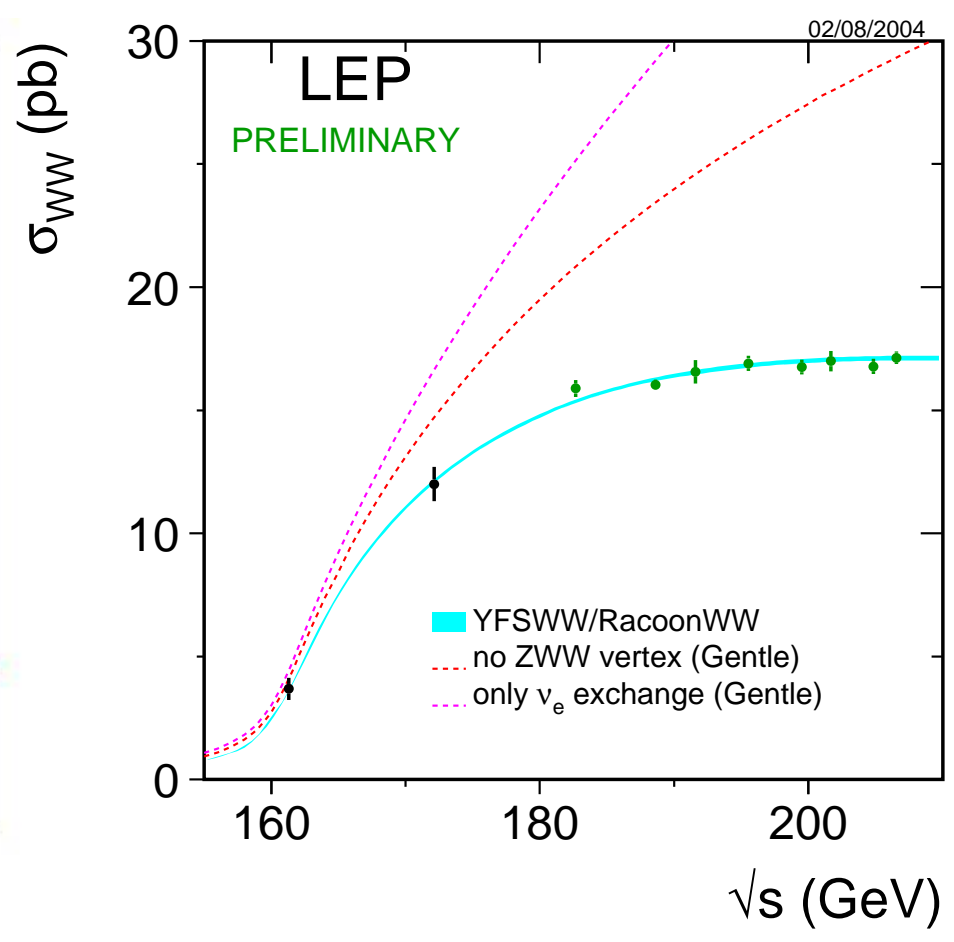

FIG. 3: ZWW contribution

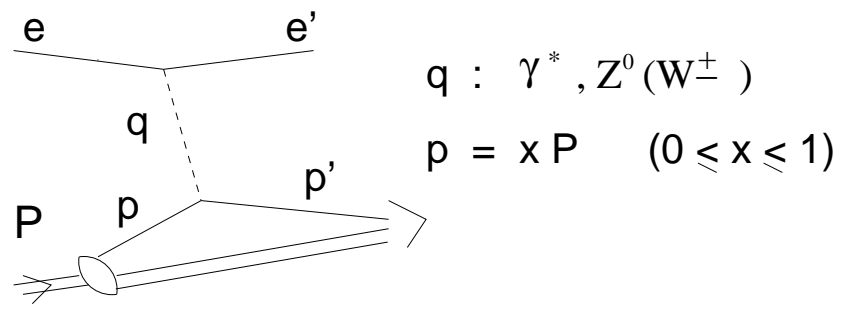

FIG. 4: DIS kinematics

That last observation conducted to the HERA idea, namely, an electron (positron) - proton collider. Electrons are accelerated up to $27.5 \mathrm{GeV}$ and protons to $920 \mathrm{GeV}$ giving rise to a center of mass energy available of around $320 \mathrm{GeV}$. In this machine, electrons (or positrons) constitute an electroweak probe to analyze the proton structure.

The deep inelastic scattering (DIS) of a lepton by a proton (at high enough $Q^{2}$ ) is seen as the elastic scattering of the lepton by a point-like constituent of the proton acting incoherently, that is called parton. In Fig. 4, the schematic diagram of the process is presented together with the relevant kinematics. Notice that $Q^{2}=-q^{2}$ can be interpreted as the resolving power of the processes.

In HERA, $Q^{2}$ runs from $4 \mathrm{GeV}$ to $40,000 \mathrm{GeV}$, corresponding to distances inside the proton ranging from $10^{-14} \mathrm{~cm}$ to $10^{-16} \mathrm{~cm}$, that means $1 / 1000$ the proton radius.

The cross section for the process is written, at the parton model level, as

$$
\sigma_{e p}=\sigma_{e q} \otimes f_{q}(x)
$$

That is the convolution of a point like electron-quark cross 
section with the probability of finding this quark into the nucleon. When QCD interactions are taken into account, the so called structure functions $f_{q}$ that measure the momentum distribution among proton constituents, acquire a $Q^{2}$ dependence. These $f\left(x, Q^{2}\right)$ have to be determined from experimental data at a given $Q_{0}^{2}$, while their dependence (evolution) with $Q^{2}$ is given by QCD dynamics. With increasing $Q^{2}$, more partons are involved with lower values of $x$ and for this reason the $f_{q}$ are larger in this region. These functions depend, in turns, on the flavor composition of the proton, namely on the distribution of the flavors $u, d, s, \bar{u}, \bar{d}, \ldots$ of quarks.

As the experimental collaborations ZEUS an H1 at HERA, were able to make precise measurements of angular distributions in DIS, it was possible to conclude that the proton, even at a very large resolution $Q^{2}$ has the valence quark structure $u, u, d$.

A very impressive result of HERA is shown in Fig. 5 where it is clear that at large momentum transfer, the cross sections of charged (via $W$ ) and neutral (via $\gamma$ and $Z$ ) currents are equal. In other words, at $Q^{2}$ sufficiently large, the electromagnetic and weak forces present the same intensity.

Other result that should be mentioned is related with the use of polarized beams. The charged current cross section measured as a function of electron (positron) polarization, allows to conclude that an eventual carrier of right handed weak interactions is excluded in all the HERA kinematic range.

Finally, let us mention that HERA provided a bound for the eventual spatial extension of quarks. The experiments found that the quark radius is smaller than $0.6710^{-3} \mathrm{fm}$.

Before leaving HERA, let us mention the main impact of its results in connection with the next proton-proton collider, LHC. This impact is related to the distribution of partons measured that are fundamental for event predictions in LHC.

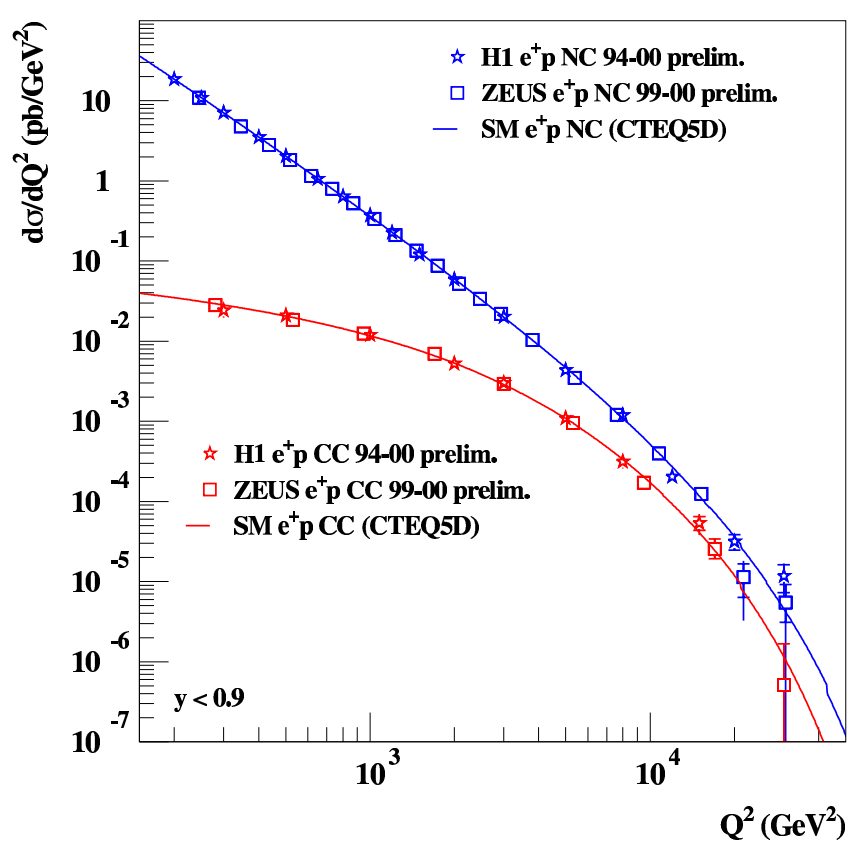

FIG. 5: Electroweak unification

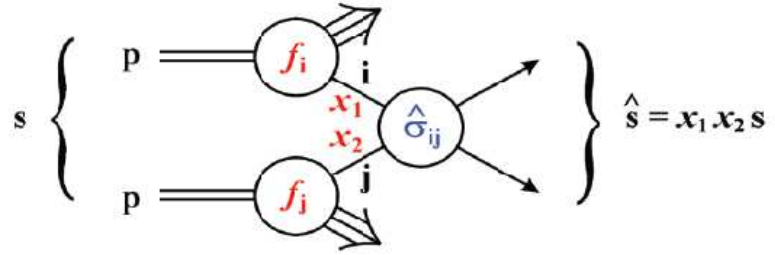

FIG. 6: pp kinematics

Moreover, the analysis of diffractive processes in HERA allows to quantify re-scattering in LHC, in order to extract clean results.

Let us go now to LHC, the Large Hadron Collider at CERN. This machine will explore energies (and consequently distances) totally new (above $1 \mathrm{TeV}$ ). Each beam of protons in LHC, accelerated inside the same tunnel where LEP was working, has an energy of $7 \mathrm{TeV}$. The main physics objectives of this collider are the search of the Higgs boson, the search of new physics as supersymmetry and large extra dimensions and the "search" of what is not searched. The main (multi-task) detectors are ATLAS and CMS. Another one, called ALICE was designed for analyzing heavy ion collision when heavy ions are accelerated instead of protons.

The first steps of the LHC project start from a clear understanding of the detectors, then follows the measurements of hadronic jets and their comparison with QCD. Calibration of energy is obviously necessary and the analyses of events with $W \mathrm{~s}$ and $Z \mathrm{~s}$ are used for check. Next is the study of top physics to finally arrive to the search for the Higgs.

Typically, the cross sections of interest look like

$$
\sigma=\sum_{i, j} \int d x_{1} d x_{2} f_{i}\left(x_{1}, Q^{2}\right) f_{j}\left(x_{2}, Q^{2}\right) \hat{\boldsymbol{\sigma}}\left(x_{1}, x_{2}, s, \alpha_{s}\right)
$$

where $\hat{\sigma}\left(x_{1}, x_{2}, s, \alpha_{s}\left(Q^{2}\right)\right)$ is calculable and the parton densities $f_{i}$ and $f_{j}$ are universal and obtained from previous experiments. The notation used in the above expression is related to the scheme of Fig. 6.

It is interesting to notice that a possible road to Higgs is the reaction $p+p \rightarrow H \rightarrow \gamma+\gamma$ presented in Fig. 7 and where all the 3 ! is fully present

In fact, this process includes the presence of gluons in the proton, the QCD running coupling constant $\alpha_{S}\left(Q^{2}\right)$, the Higgs coupling proportional to the fermion mass, the gauge coupling $W W \gamma$, the presence of virtual particles and the quantum effects present in loops. The important point is that this process is completely calculable.

Going for a while to the theoretical aspects related to LHC physics, let us begin by specifying some important topics that are beyond the Standard Model 3! One important question to be solved is related to the origin of the particle masses. Certainly the Higgs is fundamental when added to confinement but no doubt it could be something else. Next the question of unification of interactions (at, let us say, around $10^{16} \mathrm{GeV}$ ) is 
always present. The inclusion of gravity at a quantum level has to be solved even if the string trail seems to be promising. Large extra dimensions, supersymmetry, hidden sectors as unparticles, for example, are new theoretical roads to be explored.

Regarding supersymmetry remember that this bosonfermion symmetry predicts the existence of a new world of particles with masses in the rank of the $\mathrm{TeV}$, the supersymmetric mates of the known particles. These new particles have the same quantum numbers as the ordinary ones, but their spins differ in $1 / 2$ and certainly have different masses because they have not appeared yet. The spectrum is enlarged by including gauginos $(s=1 / 2)$ : wino, zino, gluino, fotino; the sparticles $(s=0)$ : squarks, sleptons; and also Higgsinos, gravitinos,...

In connection with superparticles, one can define de so called $R$ - parity by

$$
R=(-1)^{3 B+L+2 S}
$$

where $B$ is the baryon number, $L$ the lepton number and $S$ the spin. Normal particles have $R=+1$ and the supersymmetric partners $R=-1$. Certainly, if $B, L$ and $S$ are conserved so is $R$ - parity and consequently, the lightest supersymmetric particle $(R=-1)$ should be stable. This property makes this particle a good candidate for dark matter.

Supersymmetry has been around us for more than thirty years without the appearance of any experimental signal. Nevertheless, it is an appealing possibility since this symmetry provides a stabilizing mechanism for the Higgs mass, allows a clear unification of the different interactions running couplings when they are extrapolated, via the renormalization group to a large energy scale of the order of $10^{16} \mathrm{GeV}$ (as shown in Fig 8)and as mentioned before, provides a sensible candidate for dark matter with the correct abundance and a very weak interaction.

A long standing problem for the 3 ! is the so called hierarchy problem, related to the huge difference $(\sim 16$ orders of magnitude) between the Planck scale $\left(M_{P} \sim 10^{19} \mathrm{GeV}\right)$ and the electroweak scale $(1 \mathrm{TeV})$. A possible answer to this dilemma, recently proposed (1998) is the eventual presence of large extra dimensions in the Universe. This large prop-

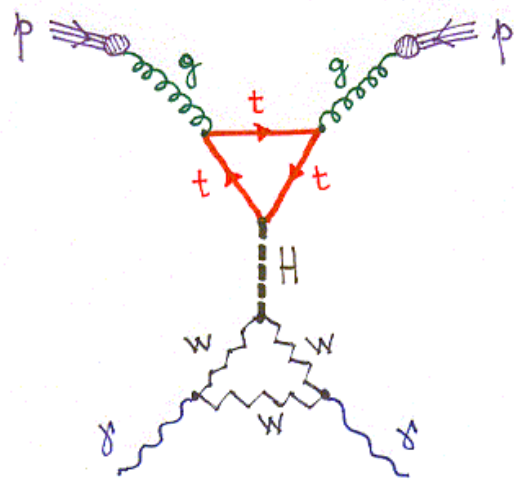

FIG. 7: Possible road to Higgs erty is so when compared with the Planck length, related to $M_{P}$ that is $l_{P}=\sqrt{\hbar G / c} \sim 1610^{-35} \mathrm{~m}$. Taking into account that the gravity interaction behavior $1 / r^{2}$ has been measured down to $\sim 1 \mathrm{~mm}$, this could be the eventual scale of the extra dimensions. Nevertheless, being the 3 ! forces sensitive to these dimensions, the actual measurements impose the bound $l_{e}<10^{-16} \mathrm{~cm}$. In the usual models of extra dimensions, ordinary matter lives in $3+1$ dimensions and in the extra ones (the bulk) only gravity works. Whenever extra dimensions are present, the Planck mass is related to these dimensions through

$$
M_{P}^{2}=M_{*}^{(2+N)} R^{n}
$$

where the volume in n dimensions $V$ is proportional to $R^{n}$, so that if $V$ is sufficiently large $\left(R \gg l_{P}\right)$, the effective Planck mass in $4+n$ dimensions could be

$$
M_{*} \ll M_{P}
$$

and if eventually $M_{*} \sim$ electroweak scale, the contact between the present particle scale and gravity could be attained.

Another spectacular effect in this scheme is the possibility of the black hole production at collider energies. In fact, inside scenarios where gravity is the protagonist at energies around $1 \mathrm{TeV}$, eventual black holes of this scale of masses could be detected. Extra dimensions makes possible this detection because here a dilution of field lines happens. The detection of black hole production via its decay goes through different steps, namely the balding (loosing of higher multipolar moments) by emission of radiation and charges; spindown, by loosing angular momentum; the next step is called Schwarzschild when the Hawking quantum radiation (thermal) is emitted. Finally the Planck step where quantum gravity is in order and almost nothing can be said.

Before ending we would like to mention a couple of very impressive recent "experiments". One is the lattice QCD calculation of the nucleon-nucleon interaction that was able to obtain the typical $N-N$ potential that includes the one pion exchange, the attractive part and the short distance core as is shown in Fig 9

The other data is related to Hubble + Chandra + Lensing experiments that detected the collision between two clusters of galaxies. The interaction of ordinary matter generated $X-$ rays detected by Chandra. Dark matter went through with almost no interaction and the invisible mass was detected by Lensing. Today one knows that dark matter is around $25 \%$ of the total stuff of the Universe and dark energy around $70 \%$, while our known material is only $5 \%$. This shows that the Universe is very different from the image of it we had ten years ago.

We include here some selected bibliography that provides a further reading guide.

http://cdsweb.cern.ch/collection/LEP

http://www.fnal.gov/pub/forphysicists/index.html

http://www-zeus.desy.de/

http://www-h1.desy.de/

http://www-hermes.desy.de/

http://lhc.web.cern.ch/lhc/ 

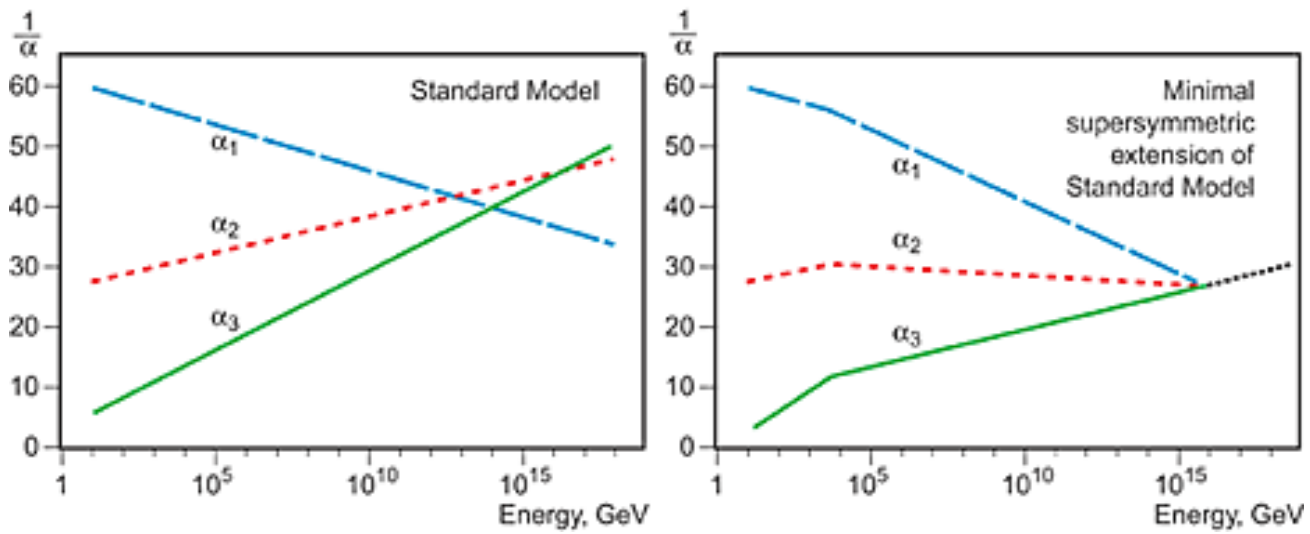

FIG. 8: Unification of interactions helped by SUSY

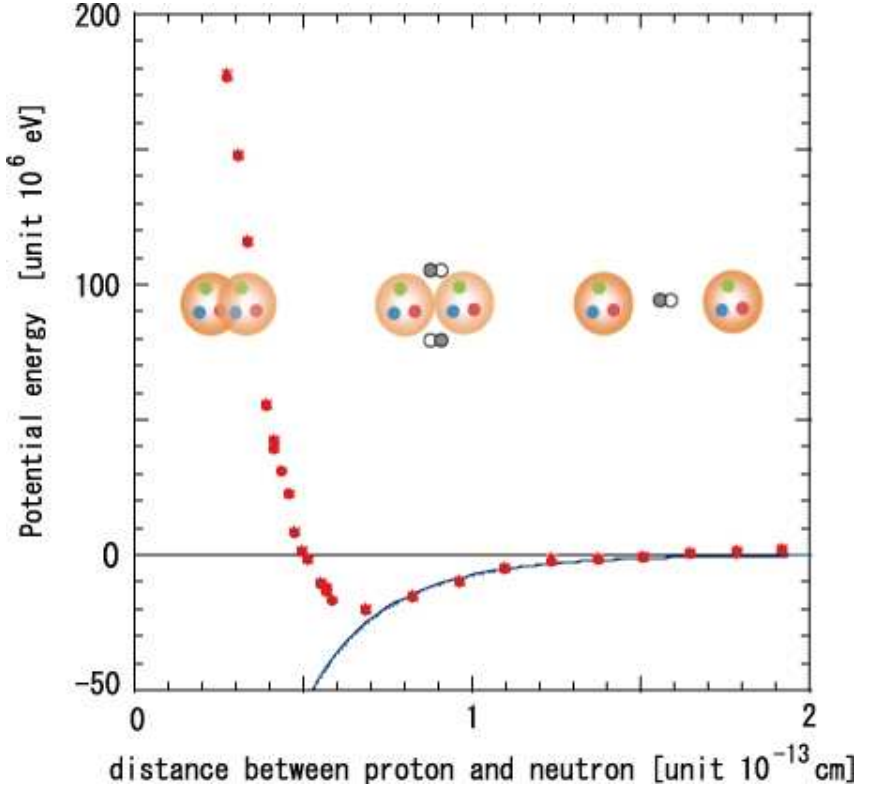

FIG. 9: Nucleon-Nucleon potential
New Physics at the LHC: A Les Houches Report. Physics at Tev Colliders 2007 - New Physics Working Group. arXiv:0802.3715v1 [hep-ph]

\section{Acknowledgements}

I would like to warmly thank Maria Beatriz Gay for the invitation to lecture at this very interesting and fruitful workshop. Thanks are also due to the Organizing Committee for their impeccable work and to T. Tarutina for her invaluable help in preparing this manuscript. Financial support from CONICET and ANPCyT of Argentina is acknowledged. 